\title{
КОММЕНТАРИИ. ДИСКУССИЯ. КРИТИКА
}

\section{Комментарий к работе М. К. Чоччи и дp. «К созданию прототипа сферического китайского волчка»}

\author{
А. А. Зобова
}

Вниманию читателей предлагается комментарий к работе М. К. Чоччи с соавторами « К созданию прототипа сферического китайского волчка» (M. C. Ciocci, B.Malengier, В. Langerock, and B.Grimonprez "Towards a Prototype of a Spherical Tippe Top", Journal of Applied Mathematics, 2012), в которой обсуждаются изложенные в статье теоретические результаты в контексте ранее опубликованных работ, по-видимому, неизвестных авторам рассматриваемой статьи.

В комментируемой работе рассматривается задача создания прототипа китайского волчка (волчка тип-топ). Волчок тип-топ - небольшая игрушка, состоящая из шарового сегмента и цилиндрической ножки на плоской части сегмента, используемой для запуска волчка с большой угловой скоростью. Положение равновесия волчка с опорой на сегмент (ножкой вверх) является устойчивым, но если волчок сильно закрутить в этом положении, то он быстро переворачивается на $180^{\circ}$, встает на ножку и в этом положении вращается некоторое время; далее скорость вращения падает и волчок постепенно возвращается к опоре на шаровой сегмент. Известно, что для того, чтобы происходил переворот волчка на ножку, его массово-инерционные характеристики должны удовлетворять некоторым условиям. Если эти эти условия не выполнены, то волчок при сколь угодно быстрой закрутке либо не переворачивается вовсе, либо переворачивается на угол, меньший $180^{\circ}$, и «застревает» на регулярной прецессии, при которой угол между осью симметрии и вертикалью оказывается ненулевым. Цель авторов работы - создать такой прототип, который при изменении расположения внутренних масс демонстрирует все три указанных варианта динамики. Такой прототип не только позволяет сравнить аналитические результаты и движение волчка

Получено 27 марта 2012 года

После доработки 27 апреля 2012 года

Зобова Александра Александровна azobova@gmail.com

Московский государственный университет им. М. В. Ломоносова 119991, Россия, г. Москва, Ленинские горы, д. 1 
в натурном эксперименте, но и, как отмечают сами авторы статьи, представляет собой интересный материал для работы со студентами.

В статье для полноты изложения представлены результаты исследования динамики простейшей модели китайского волчка. Авторами рассматривается следующая механическая модель: волчок представляет собой абсолютно твердый шар с осевой симметрией распределения масс, движущийся по неподвижной абсолютно твердой горизонтальной плоскости в поле силы тяжести. Взаимодействие между шаром и плоскостью описывается силой вязкого трения, пропорциональной скорости наинизшей точки волчка (скорости скольжения) и величине вертикальной реакции плоскости с коэффициентом трения $\mu$. Момент трения полагается равным нулю.

Авторы приводят в статье подробный вывод уравнений движения, анализ устойчивости прецессионых движений и вращений волчка с вертикально расположенной осью вращения. На основе результатов исследования устойчивости пространство массово-инерционных параметров волчка разделено на области с качественно различной динамикой, для каждой области представлена бифуркационная диаграмма. Также авторы формулируют теорему о глобальной динамике волчка тип-топ.

Анализ устойчивости вертикальных вращений и прецессионных движений волчка проводится авторами по первому приближению, причем авторы вычисляют корни характеристического уравнения в виде рядов по малому параметру $\mu$ с точностью $O\left(\mu^{2}\right)$. Повидимому, стремление вычислить корни характеристического уравнения вызвано одной из целей статьи создать интересную методическую работу для студентов, так как широко известен гораздо более изящный и эффективный метод полного нелинейного анализа устойчивости.

Существует много работ, посвященных исследованию существования и устойчивости стационарных движений тел вращения на плоскости с вязким трением. Одними из первых являются работы $[1,3,4]$ (подробную библиографию можно найти в книге [9]), в которых получены условия устойчивости вертикальных вращений неоднородного шара на плоскости с трением. Произвольные стационарные движения исследовались в работах $[2,5]$. Наиболее полный анализ существования и устойчивости стационарных движений дан в статьях А.В.Карапетяна и В.Н. Рубановского [7, 8], а также в монографии [11]. В этих работах исследование проводится на основе модифицированной теории Рауса: исследуется существование и характер критических точек эффективного потенциала - минимума полной механической энергии на фиксированном уровне интеграла Джеллетта. Одной из замечательных особенностей этого исследования является тот факт, что на вид силы трения накладываются лишь самые общие условия (диссипация энергии, равенство нулю силы трения при нулевой скорости скольжения и равенство нулю момента трения). На плоскости характерных параметров (расстояние от центра масс до геометрического центра, отношение моментов инерции) выделены области, в которых бифуркационные диаграммы имеют существенные качественные различия, для каждой области построена характерная бифуркационная диаграмма. Проведено доказательство того факта, что на вертикальных вращениях и прецессионных движениях и только на них скорость скольжения равна нулю, и, следовательно, полная механическая энергия сохраняется только на этих движениях. Таким образом, математические результаты об устойчивости и бифуркации стационарных движений, изложенные в разделах $2,4,5$ комментируемой статьи, содержатся в работах $[7,8,11]$.

Вслед за этими работами появились работы зарубежных авторов $[10,13,14]$, в которых также исследуются стационарные движения волчка тип-топ путем составления функций Ляпунова из убывающей функции энергии и интеграла Джеллетта, то есть, по существу, 
тем же методом, что и в упомянутых выше работах А. В. Карапетяна и В. Н. Рубановского. Однако надо отметить, что в работе [10] условия устойчивости вращений вокруг вертикали и прецессионных вращений волчка были получены лишь для больших значений постоянной интеграла Джеллетта. В [13] исследована устойчивость вращения волчка с вертикальным расположением оси симметрии и доказано существование переворота волчка на $180^{\circ}$ на плоскости с вязким трением (в этой статье также дана подробная библиография). В статье [14] основные результаты повторяют результаты анализа стационарных движений, представленные в [8]. В работе [15] метод Рауса также применяется для исследования устойчивости стационарных движений, однако в работе сделаны некоторые дополнительные предположения о структуре сил (авторы пренебрегают «трансляционным эффектом» - в силе трения выбрасываются члены, содержащие скорость центра масс).

Теорема о нестационарной динамике волчка при нулевом моменте трения, предлагаемая авторами, действительно сразу следует из фактов существования и устойчивости прецессионных движений и вертикальных вращений. Однако более полные результаты были получены ранее в [16]. В этой работе проведен анализ движения волчка по плоскости с ненулевой силой и моментом трения. На основе исследований устойчивости стационарных движений «невозмущенной системы» с нулевым моментом трения построены бифуркационные диаграммы Смейла - кривые стационарных движений на плоскости $\left(J^{2}, h\right)$, где $h$ - величина полной механической энергии, которая сохраняется на стационарных движениях и убывает не всех остальных. Анализируя движение изображающей точки по плоскости $\left(J^{2}, h\right)$, автор наглядно проводит полное исследование нестационарных движений волчка. Необходимо отметить также работу [6], в которой проводится исследование характера асимптотического стремления оси симметрии волчка к вертикали на горизонтальной плоскости с малым вязким трением.

Заметим, что в последнее время появились работы, посвященные анализу динамики модели волчка тип-топ, представляющего собой сферический сегмент с ножкой («двусферическая» модель волчка впервые предложена в статье [12]). Предполагается, что волчок состоит из двух шаровых сегментов, жестко связанных стержнем, направленным вдоль общей оси симметрии этих сегментов. Размеры шаровых сегментов и стержня подобраны так, что при отклонении оси симметрии от восходящей вертикали до некоторого критического значения волчок опирается о плоскость одним сегментом, а при бо́льших отклонениях другим сегментом (при критическом значении - двумя сегментами). Анализ стационарных движений такой модели волчка выполнен в работе [17], а анализ нестационарных движений с переворотом при различных моделях трения представлен в работе [18].

Таким образом, рассматриваемая статья не содержит новых теоретических результатов. Тем не менее, эта статья представляет определенный методический интерес (раздел 6 создание прототипа, раздел 7 - результаты численных исследований) и предлагает интересную для работы со студентами идею о создании прототипов различных механических систем при помощи современных технологий.

\section{Список литературы}

[1] O'Brien S., Synge J.L. The instability of the tippe-top explained by sliding friction // Proc. Roy. Irish Acad. Sect. A., 1954, vol. 56, no. 3, pp. 23-35.

[2] Румянцев В. В. Об устойчивости движения гиростатов некоторого вида // ПММ, 1961, т. 25, № 4 , c. $778-784$.

[3] Contensou P. Couplage entre frottement de glissement et frottement de pivotement dans la théorie de la toupie // Kreiselprobleme Gyrodynamics: IUTAM Symp. Celerina. Berlin: Springer, 1963. P. 201- 
216. [Контенсу П. Связь между трением скольжения и трением верчения и ее учет в теории волчка // Проблемы гироскопии. М.: Мир, 1967. С. 60-67.]

[4] Magnus K. Kreisel: Theorie und Anwendungen. Berlin: Springer, 1971. 493 S. [Магнус К. Гироскоп: теория и применение. М.: Мир, 1974. 526 с.]

[5] Карапетян А.В. О регулярной прецессии тела вращения на горизонтальной плоскости с трением // ПММ, 1982, т. 46, № 4, с. 568-572.

[6] Маркеев А. П. К динамике волчка // МТТ, 1984, № 3, с. 30-38.

[7] Карапетян А. В., Рубановский В. Н. Об устойчивости стационарных движений неконсервативных систем // ПММ, 1986, т. 50, № 1, с. 43-49.

[8] Карапетян А.В. Качественное исследование волчка на плоскости с трением // ПММ, 1991, т. 55 , № 4, с. $698-701$.

[9] Маркеев А. П. Динамика тела, соприкасающегося с твердой поверхностью. М.: Наука, 1992. $336 \mathrm{c}$.

[10] Ebenfeld S., Scheck F. A new analysis of the tippe top: Asymptotic states and Liapunov stability // Ann. Physics, 1995, vol. 243, no. 2, pp. 195-217.

[11] Карапетян А. В. Устойчивость стационарных движений. М.: Эдиториал УРСС, 1998. 165 с.

[12] Leine R.I., Gloker Ch. A set-valued force law for spatial Coulomb-Contensou friction // Eur. J. Mech. A Solids, 2003, vol. 22, pp. 193-216.

[13] Bou-Rabee N.M., Marsden J.E., Romero L.A. Tippe top inversion as a dissipation-induced instability // SIAM J. Appl. Dyn. Syst., 2004, vol. 3, no. 3, pp. 352-377.

[14] Rauch-Wojciechowski S., Sköldstam M., Glad T. Mathematical analysis of the tippe top // Regul. Chaotic Dyn., 2005, vol. 10, no. 4, pp. 333-362.

[15] Ciocci M. C., Langerock B. Dynamics of the tippe top via Routhian reduction // Internat. J. Bifur. Chaos Appl. Sci. Engrg., 2007, vol. 12, no. 6, pp. 602-614.

[16] Карапетян А. В. Глобальный качественный анализ динамики китайского волчка (тип-топ) // MTT, 2008, № 3, с. 33-41.

[17] Зобова А. А., Карапетян А. В. Анализ стационарных движений волчка тип-топ // ПММ, 2009, т. 73 , № 6 , с. $867-877$.

[18] Zobova A.A. Different models of friction in double-spherical tippe-top dynamics // IUTAM Symposium on Dynamics Modeling and Interaction Control in Virtual and Real Environments. (IUTAM Bookser., vol. 30.) Dordrecht: Springer, 2011. P. 265-272.

\section{Comments on Ciocci M. C. et al. "Towards a Prototype of a Spherical Tippe"}

Alexandra A. Zobova

Lomonosov Moscow State University

Leninskie Gory 1, Moscow, 119991, Russia

azobova@gmail.com

Received March 27, 2012, accepted April 27, 2012

Citation: Rus. J. Nonlin. Dyn., 2012, vol. 8, no. 2, pp. 427-430 (Russian) 